Article

\title{
Mechanistic Studies for Synthesis of Bis(indolyl)methanes: Pd-Catalyzed C-H Activation of Indole-Carboxylic Acids with Benzyl Alcohols in Water
}

\author{
Hidemasa Hikawa *, Hideharu Suzuki, Yuusaku Yokoyama * and Isao Azumaya \\ Faculty of Pharmaceutical Sciences, Toho University, 2-2-1 Miyama, Funabashi, Chiba 274-8510, \\ Japan; E-Mails: suzuki@phar.toho-u.ac.jp (H.S.); isao.azumaya@phar.toho-u.ac.jp (I.A.) \\ * Authors to whom correspondence should be addressed; \\ E-Mails: hidemasa.hikawa@phar.toho-u.ac.jp (H.H.); yokoyama@phar.toho-u.ac.jp (Y.Y.); \\ Tel.: +81-47-472-1591 (H.H.); Fax: +81-47-472-1595 (H.H.).
}

Received: 27 March 2013; in revised form: 9 May 2013 / Accepted: 10 May 2013 /

Published: 16 May 2013

\begin{abstract}
A method for synthesis without protecting groups of bis(indolyl)methanes by the $\left(\eta^{3}\right.$-benzyl)palladium system generated from a palladium catalyst and benzyl alcohol in water is developed. This domino protocol involves $\mathrm{C} 3-\mathrm{H}$ bond activation/benzylation of indole-carboxylic acids and benzylic $\mathrm{C}-\mathrm{H}$ functionalization. Mechanistic studies indicate that the $\left(\eta^{3}\right.$-benzyl)palladium(II) complex, which is formed via oxidative addition of benzyl alcohol 2 to a $\mathrm{Pd}(0)$ species, activates the $\mathrm{C}-\mathrm{H}$ bond at the $\mathrm{C} 3$-position of indole $\mathbf{1}$. Notably, water plays an important role in our catalytic system for $\mathrm{sp}^{3} \mathrm{C}-\mathrm{O}$ bond activation and stabilization of $\mathrm{OH}^{-}$by hydration for the smooth generation of the activated $\mathrm{Pd}(\mathrm{II})$ cation species, as well as for nucleophilic attack of indoles to hydrated benzyl alcohols.
\end{abstract}

Keywords: palladium; C-H activation; water; indole; benzyl alcohol

\section{Introduction}

The $\left(\eta^{3}\right.$-benzyl)palladium catalysts are gaining increasing interest. The powerfulness of these complexes is still far from other well-established Tsuji-Trost reactions, merely due to a more recent development [1-9]. Palladium-catalyzed benzylations with benzylic alcohols via the $\left(\eta^{3}\right.$-benzyl)palladium intermediate are especially challenging, because the reactivity of benzylic alcohols towards $\operatorname{Pd}(0)$ is poor compared to benzylic halides, esters, carbonates and phosphates. 
Therefore, the development of a direct catalytic substitution of benzylic alcohols, which produces the desired products along with water as the sole co-product, is highly desired in organic chemistry. Recently, direct application of benzyl alcohols as electrophiles in various reactions was achieved via Brønsted/Lewis acid [10-12], transition metal [13,14] or water-promoted [15-17] $\mathrm{sp}^{3} \mathrm{C}-\mathrm{O}$ bond activation.

We have developed a unique strategy for benzylation and $\mathrm{C}-\mathrm{H}$ activation [18-24] by the $\left(\eta^{3}\right.$-benzyl)palladium system from a palladium catalyst and benzyl alcohol in water [25-28]. Water activates the benzyl alcohol via hydration of the hydroxyl group for generation of the $\left(\eta^{3}\right.$-benzyl)palladium species, which can then undergo innovative direct transformation reactions. We became interested in further expanding the substrate scope of the $\left(\eta^{3}\right.$-benzyl)palladium system to water-soluble unprotected indole carboxylic acids $\mathbf{1}$, since we have been studying the development of synthesis without protecting groups and selective reactions towards various reactive functional groups [29-32]. In general, synthesis without protecting groups represents a distinct challenge and has been fraught with a number of difficulties such as chemoselectivity [33]. One of the most effective ways for achieving synthesis without protecting groups is the development of selective reactions towards various reactive functional groups. Although the possibility of generating by the different coupling reactions the undesired products shown in parentheses exists, in our catalytic system, only the desired products were obtained selectively in excellent to good yields (Scheme 1). Oxygen nucleophilicity of the carboxyl group may be weak due to hydration under aqueous conditions.

Scheme 1. Our previous work.
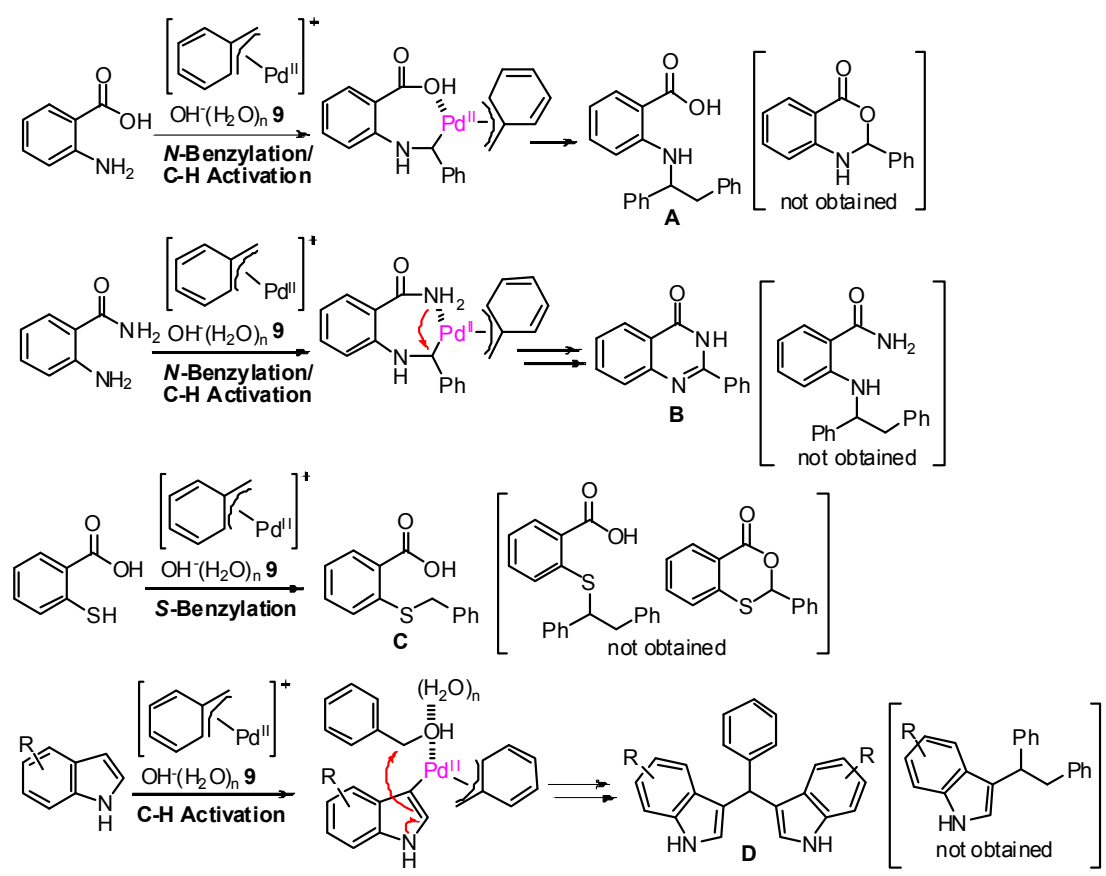

In our previous paper [25], we reported a method for the synthesis of bis(indolyl)methanes via palladium-catalyzed domino reactions of indoles with benzyl alcohols in water and suggested a plausible mechanism for the formation of bis(indolyl)methanes. In the present study, we explore the synthesis without protecting groups of bis(indolyl)methanes $\mathbf{3}$ from indole-carboxylic acids $\mathbf{1}$ and propose a more detailed mechanism based on various control experiments. Herein, we report the 
development of synthesis without protecting groups of bis(indolyl)methanes $\mathbf{3}$ via palladium-catalyzed domino reactions of indole-carboxylic acids 1 with benzyl alcohols $\mathbf{2}$ in water. Based on observations made in this investigation, we can now provide strong support for the $\mathrm{Pd}$-catalyzed $\mathrm{C} 3-\mathrm{H}$ activation and benzylation pathway. This paper describes mechanistic investigations aimed at providing a rational explanation for the formation of bis(indolyl)methanes 3 .

\section{Results and Discussion}

First, we heated a mixture of indole-5-carboxylic acid 1a and benzyl alcohol 2a (3 equiv) in the presence of $\mathrm{Pd}(\mathrm{OAc})_{2}(5 \mathrm{~mol} \%)$ and sodium diphenylphosphinobenzene-3-sulfonate (TPPMS, $10 \mathrm{~mol} \%$ ) in water at $120{ }^{\circ} \mathrm{C}$ for $16 \mathrm{~h}$ in a sealed tube. Bis(indolyl) product 3a was obtained in $84 \%$ yield along with $\mathrm{C} 3$-benzylated $\mathbf{4 a}$ in $15 \%$ yield (Table 1, entry 1). Importantly, the reaction was completely $\mathrm{C} 3$-selective, with no $\mathrm{C} 2$ - or $\mathrm{N}$-benzylated product formed. When 1a was consumed completely at $60{ }^{\circ} \mathrm{C}$ in $16 \mathrm{~h}$, the reaction afforded only desired 3a in excellent yield (entry 2, 91\%). In contrast, the reaction at room temperature did not occur (entry 3). The reaction also did not proceed in the absence of the palladium catalyst and phosphine ligand (entry 4). With regard to the palladium catalyst, the use of $\mathrm{PdCl}_{2}$ or $\mathrm{Pd}_{2}(\mathrm{dba})_{3}$ also gave the product 3a in excellent yields (entry 5, 91\%; entry 6, 93\%). Since the reaction did not occur when using $\mathrm{PdCl}_{2}\left(\mathrm{PPh}_{3}\right)_{2}$ instead of a water-soluble ligand (entry 7) or when using DMSO, EtOH or THF (entry 8) as a solvent, water must play an important role in our catalytic system. AcOH also resulted in excellent yield (entry 9, 93\%). To our surprise, the reaction proceeded when $1 \mathrm{M} \mathrm{NaOH}$ aq. was used as a solvent at $60{ }^{\circ} \mathrm{C}$ (entry 10). In our previous work, benzylation of anthranilic acid did not occur at $120{ }^{\circ} \mathrm{C}$ in $1 \mathrm{M} \mathrm{NaOH}$ aq [28]. Thus, the $\left(\eta^{3}\right.$-benzyl)palladium intermediate might be unstable under high temperature and strong basic conditions [34].

Results for the reaction of indole-5-carboxylic acid 1a with a number of benzyl alcohols $\mathbf{2}$ or several indole carboxylic acids 1 with benzyl alcohol 2a using $\mathrm{Pd}(\mathrm{OAc})_{2}$ and TPPMS are summarized in Figure 1. The benzyl alcohols with electron-donating methyl, ethyl and methoxy groups resulted in moderate to good yields (3b, 83\%; 3c, 92\%; 3d, 54\%; 3e, 54\%). The reaction of 4-fluorobenzyl alcohol also proceeded (3f, 52\%). The benzyl alcohol with a chloro group produced desired $\mathbf{3 g}$ in good yield with the carbon-halogen moiety left intact, which could be employed for further manipulation (3g, 67\%). A heteroaryl methyl alcohol, thienyl methanol, also resulted in a moderate yield (3h, 52\%). To our surprise, aliphatic benzyl alcohol, 2-naphthalenemethanol, which is not very soluble in water, gave desired product 3i in 96\% yield. The indoles with 4-, 6- and 7-carboxyl groups resulted in moderate to good yields $(3 \mathbf{j}, 52 \%$; 3k, 60\%; 31, 76\%). Interestingly, the indole with 2-carboxyl groups was also tolerated in the reaction $(\mathbf{3 m}, 45 \%)$, despite the weak nucleophilicity at the $\mathrm{C} 3$ position of indole due to the electron-withdrawing 2-carboxyl acid group. Therefore, synthesis without protecting groups is possible to prepare $\mathbf{3 m}$ with our catalytic system.

We monitored the $\mathrm{C} 3-\mathrm{H}$ activation reaction by ${ }^{1} \mathrm{H}$ NMR spectroscopy (Table 2). In general, a $\mathrm{C}-\mathrm{H}$ bond activation mechanism is used to describe the substitution of indoles by electrophilic metals such as $\mathrm{Pd}(\mathrm{II})$ [35-38]. Thus, indole 1a reacted with ( $\eta^{3}$-benzyl)palladium(II) to form intermediate $\mathbf{A}$, followed by formation of intermediate $\mathbf{B}$, which reacted with $\mathrm{D}_{2} \mathrm{O}$ to give C3-D indole 1a' (Scheme 2, path A). Indeed, treatment of indole-5-carboxylic acid 1a with $\operatorname{Pd}_{2}(\mathrm{dba})_{3}$, TPPMS and benzyl alcohol 
2a in $\mathrm{D}_{2} \mathrm{O}$ at $60{ }^{\circ} \mathrm{C}$ for $3 \mathrm{~h}$ showed $65 \%$ deuterium incorporation at the $\mathrm{C} 3$-position of indole (entry 1 ). In contrast, in the absence of benzyl alcohol $\mathbf{2 a}$, or when using toluene instead of $\mathbf{2 a}$, only a trace amount of deuterium was incorporated (entry $2 ; 12 \%$, entry 3; 14\%). Use of 4-methylbenzyl alcohol $\mathbf{2 b}$ or 4-chlorobenzyl alcohol $\mathbf{2 c}$ instead of $\mathbf{2 a}$ resulted in good yields (entry 4; 80\%, entry 5; 70\%). These results suggested that the palladium(II) complex which was formed via oxidative addition of benzyl alcohol 2 to a $\mathrm{Pd}(0)$ species activated the $\mathrm{C}-\mathrm{H}$ bond at the $\mathrm{C} 3$-position of 1a. While the indole with 2-carboxylic acid ethyl ester $\mathbf{1 b}$ resulted in no reaction (entry $6 ; 3 \%$ ), the indole with 2-carboxylic acid 1c showed $81 \%$ deuterium incorporation (entry 7). This observation suggested that the 2-carboxylic acid moiety plays an important role as a directing group in $\mathrm{C} 3-\mathrm{H}$ palladation to afford the desired product $\mathbf{3 m}$ (Figure 2). In addition, 1,2-migration of intermediate $\mathbf{A}$ did not occur to give C2-palladated C (Scheme 2, path B).

Table 1. Effect of catalysts and solvents ${ }^{\mathrm{a}}$.

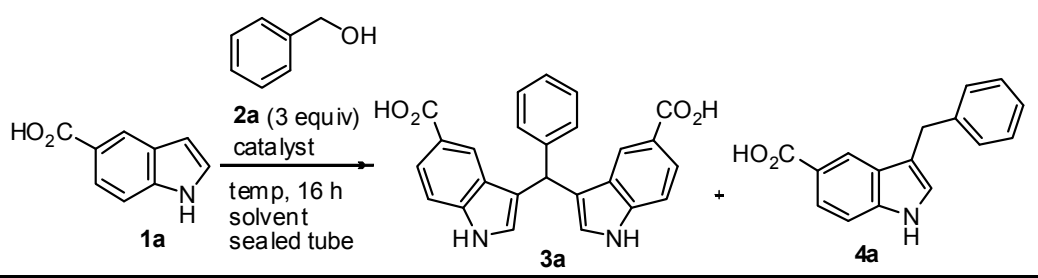

\begin{tabular}{llllll}
\hline Entry & Catalyst & Solvent & \multicolumn{2}{c}{ Temp } & \multicolumn{2}{c}{ product $(\%))^{\mathbf{b}}$} \\
& & & $\left({ }^{\circ} \mathbf{C}\right)$ & 3a & 4a \\
\hline 1 & $\mathrm{Pd}(\mathrm{OAc})_{2} /$ TPPMS & $\mathrm{H}_{2} \mathrm{O}$ & 120 & 84 & 15 \\
2 & $\mathrm{Pd}(\mathrm{OAc})_{2} / \mathrm{TPPMS}$ & $\mathrm{H}_{2} \mathrm{O}$ & 60 & $91(92)^{\mathrm{c}}$ & trace \\
3 & $\mathrm{Pd}(\mathrm{OAc})_{2} / \mathrm{TPPMS}$ & $\mathrm{H}_{2} \mathrm{O}$ & $\mathrm{rt}$ & trace & 0 \\
4 & none & $\mathrm{H}_{2} \mathrm{O}$ & 60 & 0 & 0 \\
5 & $\mathrm{PdCl}_{2} / \mathrm{TPPMS}$ & $\mathrm{H}_{2} \mathrm{O}$ & 60 & 91 & trace \\
6 & $\mathrm{Pd}_{2}(\mathrm{dba})_{3}{ }^{\mathrm{d}} / \mathrm{TPPMS}$ & $\mathrm{H}_{2} \mathrm{O}$ & 60 & 93 & trace \\
7 & $\mathrm{PdCl}_{2}\left(\mathrm{PPh}_{3}\right)_{2}$ & $\mathrm{H}_{2} \mathrm{O}$ & 60 & trace & trace \\
8 & $\mathrm{Pd}(\mathrm{OAc})_{2} / \mathrm{TPPMS}$ & $\mathrm{DMSO}, \mathrm{EtOH}$ or THF & 60 & 0 & 0 \\
9 & $\mathrm{Pd}(\mathrm{OAc})_{2} / \mathrm{TPPMS}$ & $\mathrm{AcOH}$ & 60 & 93 & trace \\
10 & $\mathrm{Pd}(\mathrm{OAc})_{2} / \mathrm{TPPMS}$ & $1 \mathrm{M} \mathrm{NaOHaq}$. & 60 & 63 & trace \\
\hline
\end{tabular}

${ }^{a}$ Reaction conditions: 1a $(0.5-1 \mathrm{mmol})$, Pd catalyst (5 mol\%), ligand (10 mol\%), benzyl alcohol 2a (3 equiv), solvent $(1-2 \mathrm{~mL}), \mathrm{rt}-120{ }^{\circ} \mathrm{C}, 16 \mathrm{~h}$ in a sealed tube. ${ }^{\mathrm{b}}$ Yield of isolated product. ${ }^{\mathrm{c}}$ The yield was determined by ${ }^{1} \mathrm{H}$ NMR analysis of the crude product using $p$-nitroanisole as an internal standard. ${ }^{\mathrm{d}} 2.5 \mathrm{~mol} \%$. ${ }^{\mathrm{e}} 4$ equiv were used.

Figure 1. Scope of alcohols 2 and indole carboxylic acids $1{ }^{\text {a }}$ a ${ }^{\text {a }}$ Reaction conditions: 1a (0.5-1 mmol), $\mathrm{Pd}(\mathrm{OAc})_{2}$ (5 mol\%), TPPMS (10 mol\%), benzyl alcohols 2 (3 equiv), $\mathrm{H}_{2} \mathrm{O}$ $(2-4 \mathrm{~mL}), 60{ }^{\circ} \mathrm{C}, 16 \mathrm{~h}$ in a sealed tube. Yield of isolated product. ${ }^{\mathrm{b}}$ benzyl alcohols 2 (5 equiv), $80^{\circ} \mathrm{C}$.

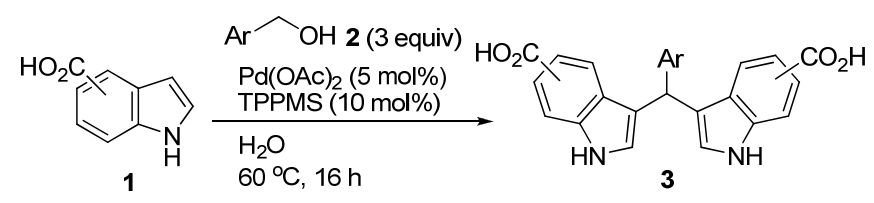


Figure 1. Cont.<smiles>CCc1ccc(C(c2c[nH]c3ccc(C(=O)O)cc23)c2c[nH]c3ccc(C(=O)O)cc23)cc1</smiles>

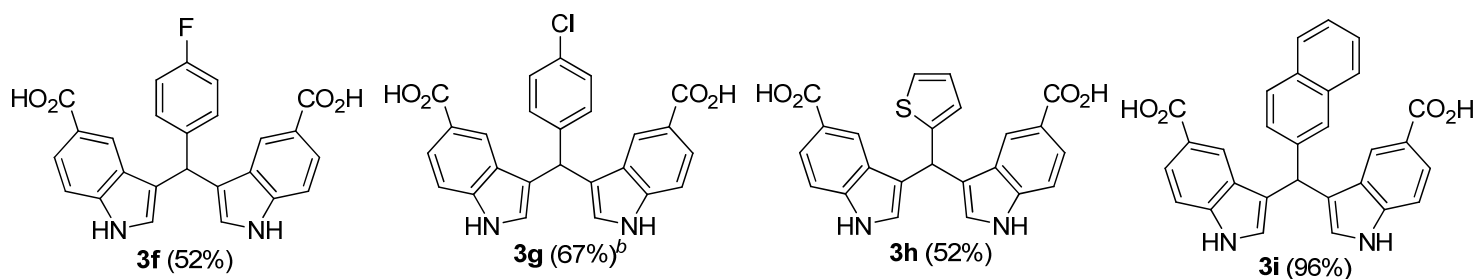

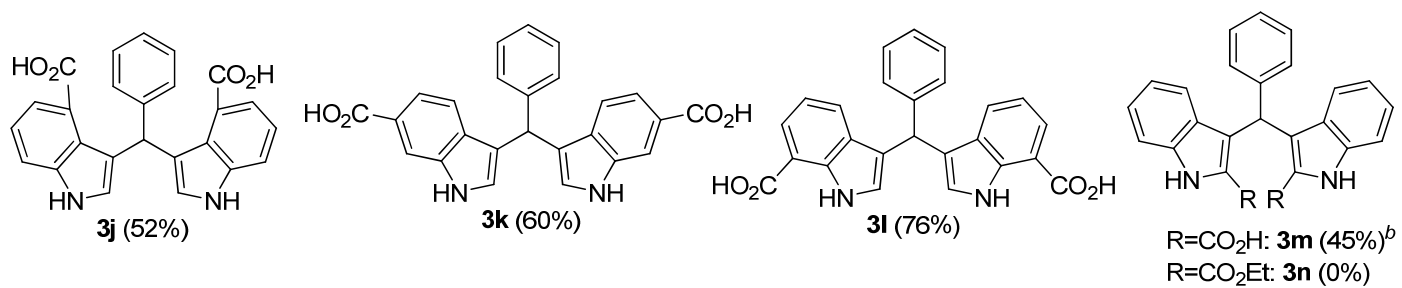

Scheme 2. Possible mechanism of $\mathrm{C}-\mathrm{H}$ bond activation.

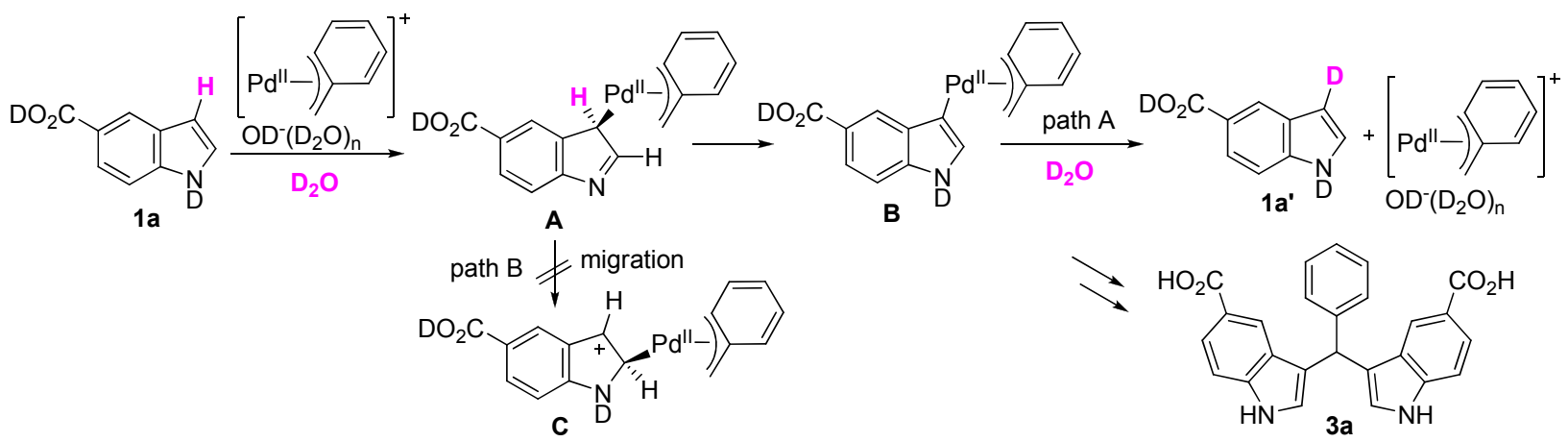

Table 2. $\mathrm{C}-\mathrm{H}$ bond activation at the $\mathrm{C} 3$-position of indole-5-carboxylic acid $\mathbf{1 a}{ }^{\mathrm{a}}$.<smiles>[R]c1ccc(CO)cc1</smiles>

\begin{tabular}{|c|c|c|c|}
\hline Entry & Indoles 1 & Benzylic alcohols 2 & D incorporation $(\%)^{b}$ \\
\hline 1 & & $\mathrm{O}_{\mathbf{2 a}}$ & 65 \\
\hline 2 & $1 \mathbf{a}$ & none & 12 \\
\hline
\end{tabular}


Table 2. Cont.

(a)

${ }^{\mathrm{a}}$ Reaction conditions: $1(0.25 \mathrm{mmol}), \mathrm{Pd}_{2}(\mathrm{dba})_{3}(2.5 \mathrm{~mol} \%)$, TPPMS (10 mol\%), benzyl alcohols 2 (3 equiv), $\mathrm{D}_{2} \mathrm{O}(0.75 \mathrm{~mL}), 60{ }^{\circ} \mathrm{C}, 3 \mathrm{~h}$ under $\mathrm{Ar}$ in a sealed tube. ${ }^{\mathrm{b}} \mathrm{D}$ incorporation was calculated by NMR integration.

Figure 2. Role of carboxylic acid as a directing group.<smiles>C[Pb](C)(O)c1c(C(=O)O)[nH]c2ccccc12</smiles>

To confirm that 3-benzylindole 4a was not the intermediate in our catalytic system, we tested the reaction of $\mathbf{4 a}$ (Scheme 3, A). The reaction afforded desired 3a (71\% from 1a) and recovery of 3-benzylated 4a (90\%). Use of 3-benzylindole $\mathbf{4 b}$ instead of $\mathbf{4 a}$ also resulted in recovery of 3-benzylated 4b (90\%) (Scheme 3, B). These results suggested that 3-benzylated 4a is not the intermediate in our catalytic system.

Scheme 3. Pd-catalyzed benzylation of indole 1a and 3-benzylindole 4.
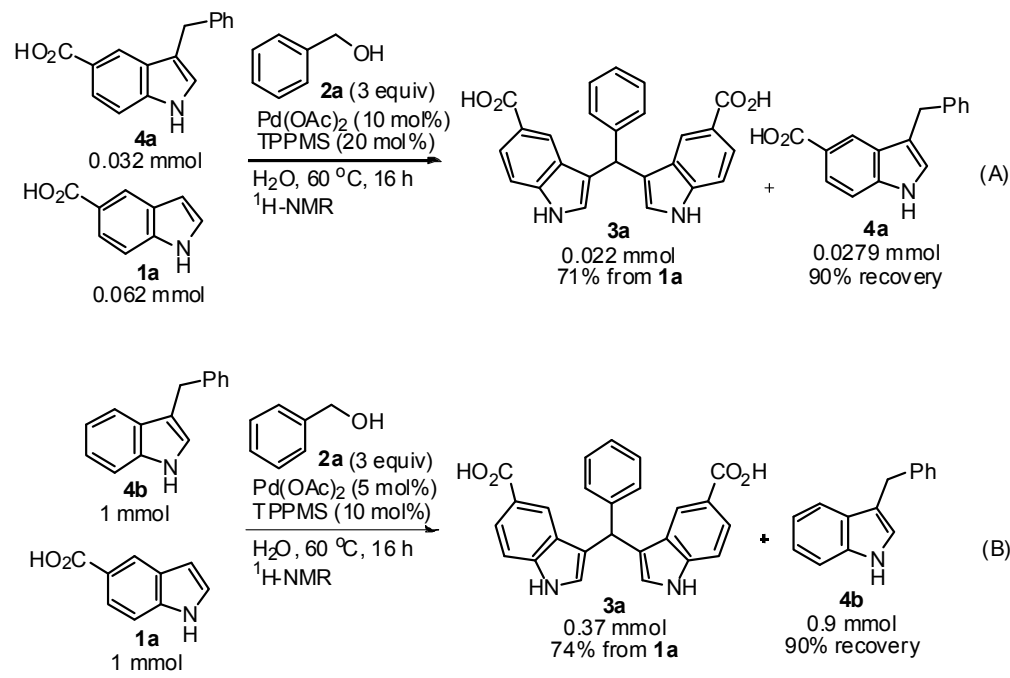
These results and our previous report [25-28] suggest the following mechanism for the formation of bis(indolyl)methanes $\mathbf{3}$ from indole carboxylic acid $\mathbf{1}$ and benzyl alcohol $\mathbf{2}$ in water (Scheme 4). Notably, activation of the $\mathrm{C}-\mathrm{H}$ bond at the $\mathrm{C} 3$-position of indole carboxylic acid $\mathbf{1}$ with ( $\eta^{3}$-benzyl)palladium 7 occurred to generate intermediate 8, which did not form 3-benzylated 4 through reductive elimination (see Scheme 3). Instead, intermediate 8 reacted with benzyl alcohol $\mathbf{2}$ to give C3-benzylated $\mathbf{1 0}$.

Scheme 4. Proposed mechanism.

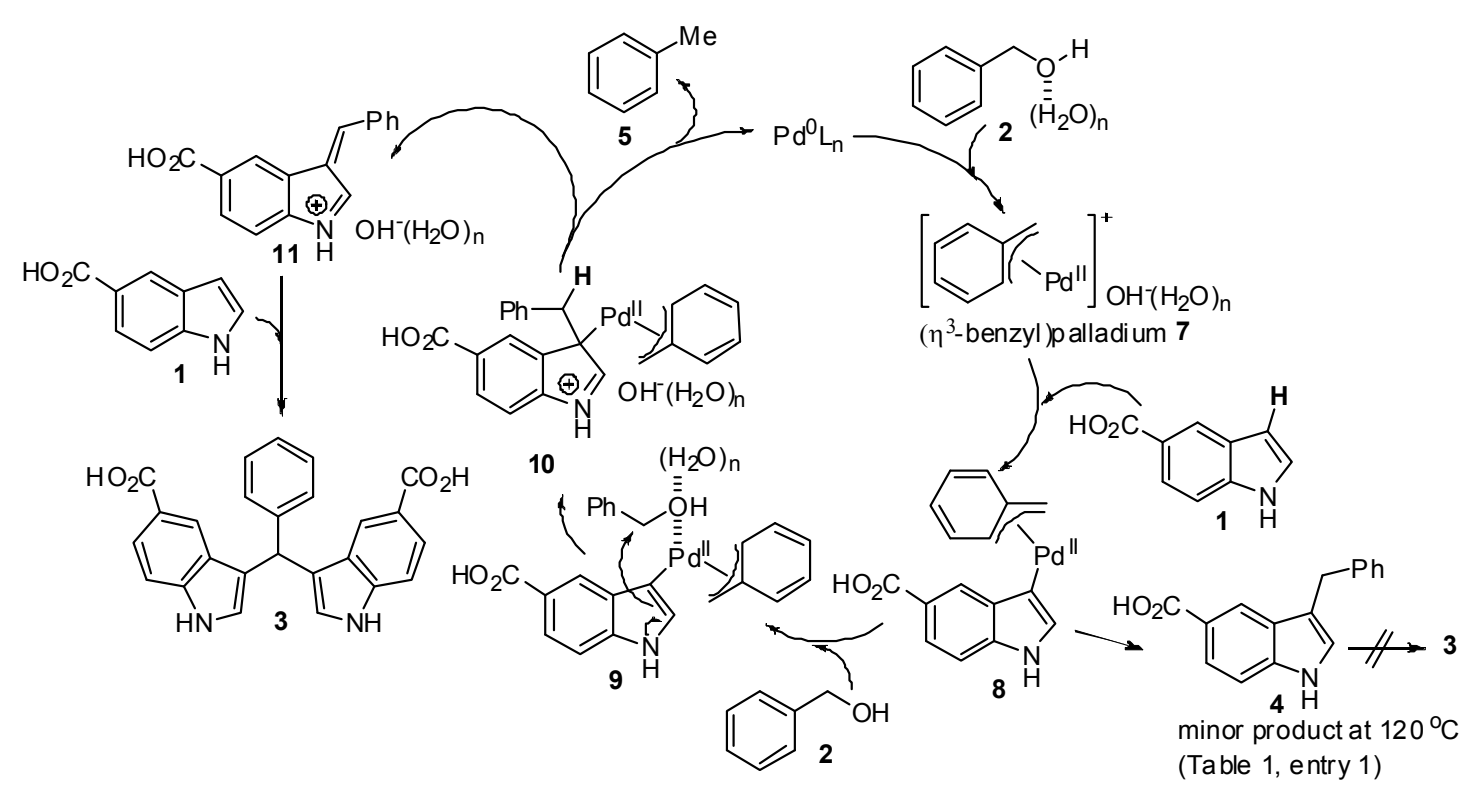

Furthermore, toluene 5 should be formed from the ( $\eta^{3}$-benzyl)palladium complex through reductive elimination in our catalytic system (see Scheme 4). We were delighted to observe that indeed toluene 5 was obtained in the reaction mixture (Sceme 5, A). In contrast, the reaction of benzyl alcohol 2a in the absence of indole 12 gave benzaldehyde 14 (14\%) and recovery of SM 2a (68\%) with no toluene 5 detected (Scheme 5, B). This observation suggested that benzaldehyde 14 should not be the intermediate in our catalytic system.

Scheme 5. ${ }^{1} \mathrm{H}$ NMR experiments to monitor the reaction.

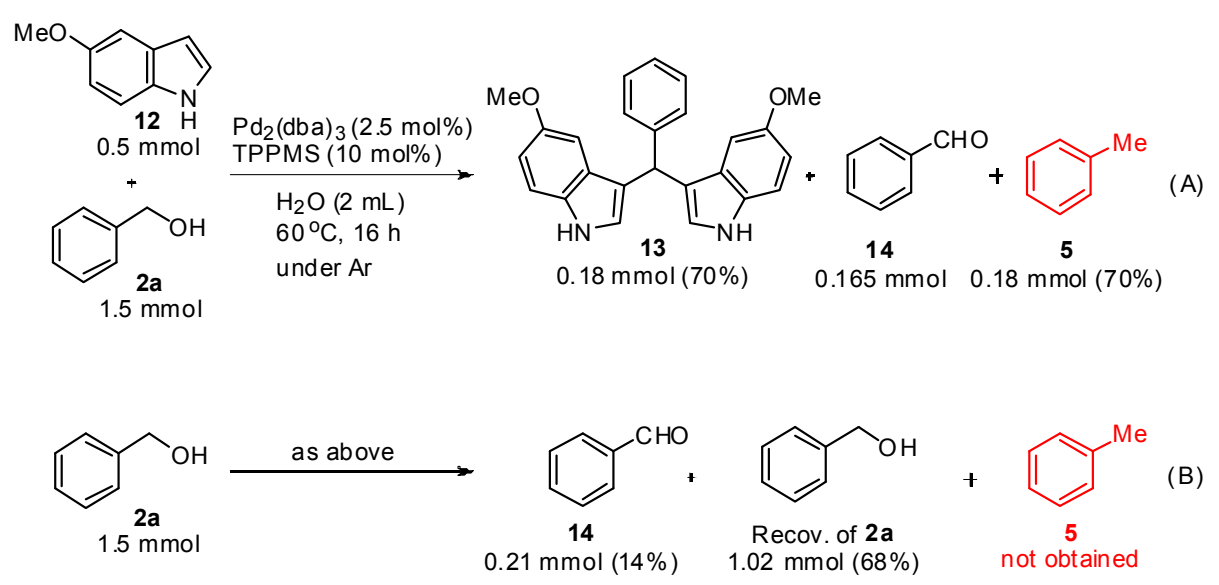


Next, we utilized ${ }^{1} \mathrm{H}$ NMR experiments to monitor the reaction using benzyl- $\alpha, \alpha-d_{2}$ alcohol 15. The reaction afforded desired deuterated $20(\mathrm{D} / \mathrm{H}=96: 4)$ and deuterated toluene $21(\mathrm{D} / \mathrm{H}=73: 27)$ (Scheme 6). This observation clearly explained that $\beta$-D elimination of intermediate 17 occurred to generate intermediate 18, followed by addition of indole 12 to give desired deuterated 20 along with deuterated toluene $\mathbf{2 1}$ with regeneration of $\operatorname{Pd}(0)$ through reductive elimination. $\beta$

Scheme 6. Pd-catalyzed reaction with benzyl- $\alpha, \alpha-d_{2}$ alcohol 15.
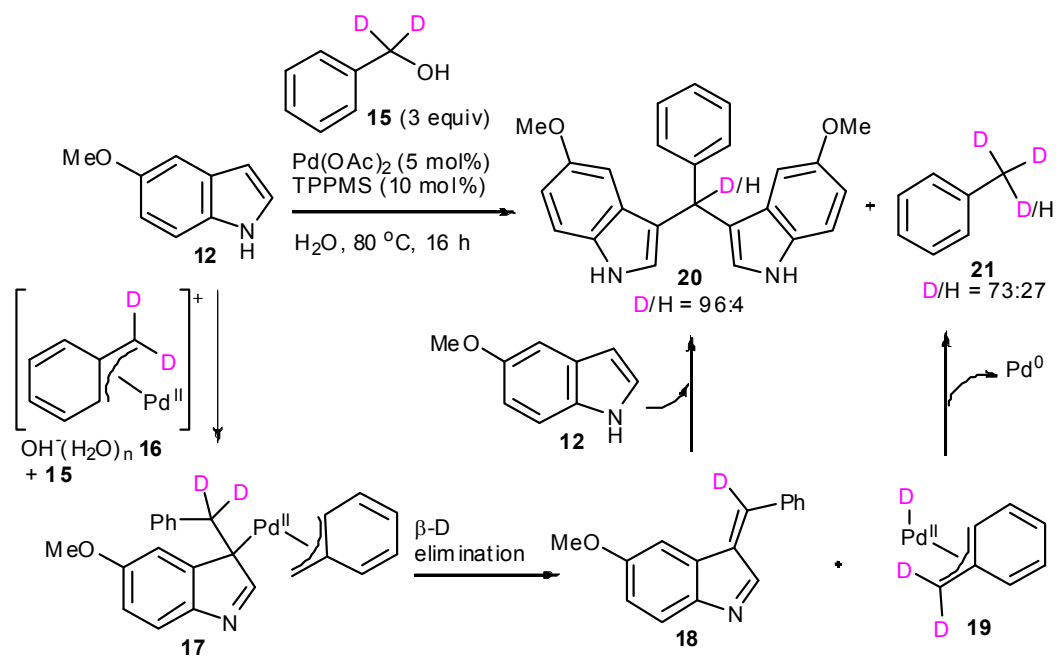

Finally, we investigated the reaction using benzyl acetate 22 instead of benzyl alcohol $\mathbf{2}$ because oxidative addition of the benzylic ester to a $\mathrm{Pd}(0)$ species could occur to form ( $\eta^{3}$-benzyl)palladium 7 $[39,40]$. As expected, 65\% deuterium incorporation was observed using benzyl acetate 22 in $\mathrm{D}_{2} \mathrm{O}$ (Table 3, entry 1). To our surprise, the reaction did not afford desired product 3a. These results suggested that the C3-palladated indole could not react with benzyl acetate $\mathbf{2 2}$ (Figure 3, intermediate 23). It is known that the reactivity of benzyl acetate 22 with nucleophiles is high compared with benzyl alcohols 2. Next, the reactions using benzyl acetate $\mathbf{2 2}$ and benzyl alcohol 2a in organic solvents such as 1,4-dioxane or $\mathrm{CD}_{3} \mathrm{OD}$ instead of water were examined, which also did not afford desired product 3a (entry 2 and 3). Interestingly, $89 \%$ deuterium incorporation was observed when $\mathrm{CD}_{3} \mathrm{OD}$ was used. These results suggested that $\mathrm{C} 3$-palladated indole could not react with benzyl alcohol 2a after $\mathrm{C}-\mathrm{H}$ activation of indole 1 occurred in $\mathrm{CD}_{3} \mathrm{OD}$, and organic solvents could not activate the benzyl alcohol 2a (Figure 3, intermediate 24). Therefore, we have demonstrated an important role of water for water-promoted $\mathrm{sp}^{3} \mathrm{C}-\mathrm{O}$ bond activation of benzyl alcohols 2 in our catalytic system (Figure 3, intermediate 9) [15-17].

This domino process includes $\mathrm{C}-\mathrm{H}$ activation/benzylation at the $\mathrm{C} 3$-position of the indole-carboxylic acid and benzylic $\mathrm{C}-\mathrm{H}$ functionalization. $\mathrm{C} 3$-Benzylation of unprotected indole carboxylic acids is extremely rare. Carter and co-workers reported the only reaction of indole-5-carboxylic acid with 4-methoxybenzylbromide using EtMgBr to afford 3-benzylated indole-5-carboxylic acid [41]. Synthesis of bis(indolyl)methanes having carboxylic acids from benzyl alcohol and indole is also extremely rare. Itoh and co-workers reported the only one-pot synthesis from indole-3-butyric acid with benzyl alcohols using catalytic iodine and oxygen under visible light 
irradiation [42]. Indole carboxylic acids and their analogs are key units in a wide range of relevant pharmacophores with a broad spectrum of activities [41-46].

Table 3. Use of benzyl acetate $12^{\mathrm{a}}$.

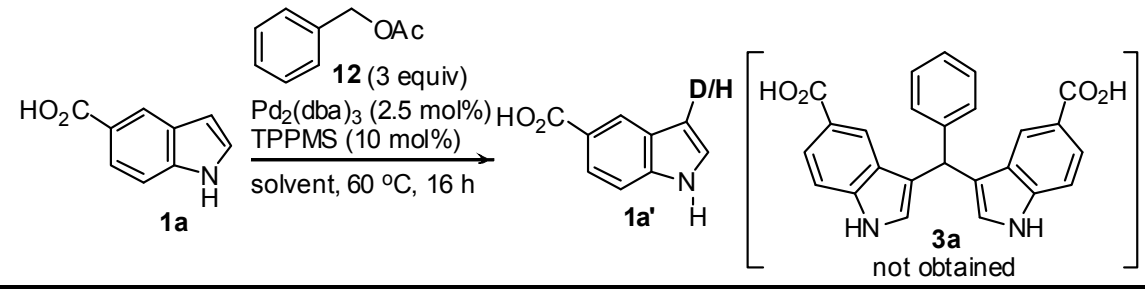

\begin{tabular}{cccc}
\hline Entry & Solvent & Additive & D incorporation (\%) $^{\mathbf{b}}$ \\
\hline 1 & $\mathrm{D}_{2} \mathrm{O}$ & none & 65 \\
2 & 1,4-dioxane & $\begin{array}{c}\text { benzyl alcohol 2a } \\
(3 \text { equiv) } \\
\text { benzyl alcohol 2a } \\
(3 \text { equiv })\end{array}$ & - \\
3 & $\mathrm{CD}_{3} \mathrm{OD}$ & 89 \\
\hline
\end{tabular}

${ }^{\mathrm{a}}$ Reaction conditions: 1a $(0.5 \mathrm{mmol}), \mathrm{Pd}_{2}(\mathrm{dba})_{3}(2.5 \mathrm{~mol} \%)$, TPPMS (10 mol\%), benzyl acetate 12 (3 equiv), solvent $(1.5 \mathrm{~mL}), 60^{\circ} \mathrm{C}, 16 \mathrm{~h}$ in a sealed tube. ${ }^{\mathrm{b}} \mathrm{D}$ incorporation was calculated by NMR integration.

Figure 3. Role of water for water-promoted $\mathrm{sp}^{3} \mathrm{C}-\mathrm{O}$ bond activation of benzyl alcohols 2 .
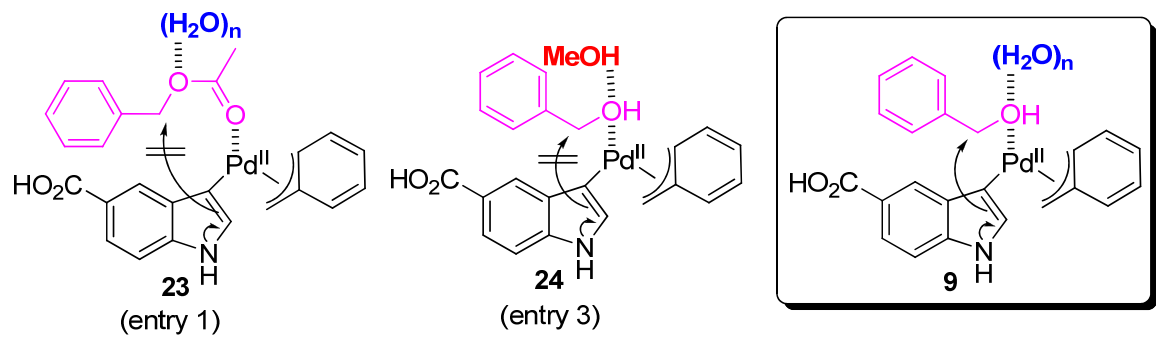

Scheme 7. Development of unprotected syntheses, new activated catalysts, and new reaction fields.

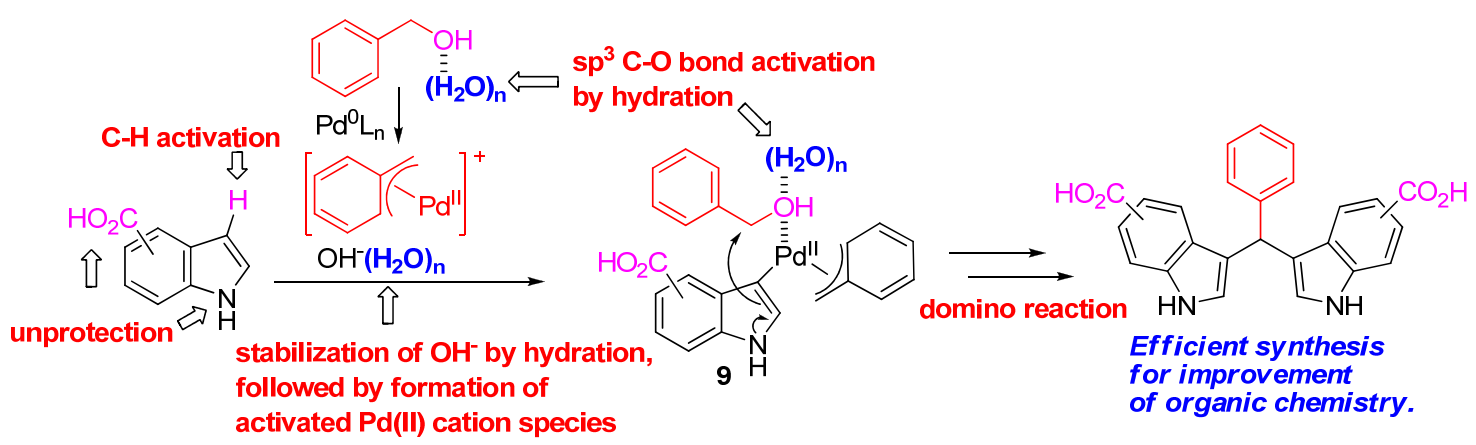

In general, almost all of the compounds that have been synthesized based on protection/deprotection steps and known organic transformations tend to be inefficient in organic solvents. To enable efficient synthesis for improvement of organic chemistry, it is essential to develop synthesis without protecting groups, newly activated catalysts that work for activating unreactive bonds such as $\mathrm{C}-\mathrm{H}$ bonds, and new reaction fields. In our catalytic system, water plays an important 
role in activation of unreactive molecules and stabilization of hydroxide ion by hydration, followed by formation of activated $\mathrm{Pd}$ (II) cation species [47]. Nucleophilic attack of indoles to hydrated benzyl alcohols also could occur (Scheme 7). In organic solvents, elimination of naked hydroxide ion is unfavorable. Thus, our catalytic system should have a broad impact on Pd-catalyzed reactions.

\section{Experimental Section}

General procedure: A mixture of indole carboxylic acid 1 (0.5-1 mmol), palladium(II) acetate (5 mol\%), sodium diphenylphosphinobenzene-3-sulfonate (TPPMS, $10 \mathrm{~mol} \%$ ) and benzyl alcohol 2 (3-5 equiv) in $\mathrm{H}_{2} \mathrm{O}(2-4 \mathrm{~mL})$ was heated at $60-80{ }^{\circ} \mathrm{C}$ for $16 \mathrm{~h}$ in a sealed tube. After cooling, the reaction mixture was poured into water $(50 \mathrm{~mL})$ and extracted with EtOAc $(100 \mathrm{~mL} \times 3)$. The organic layer was washed with brine, dried over $\mathrm{MgSO}_{4}$ and concentrated in vacuo. The residue was purified by flash column chromatography (silica gel, hexanes/EtOAc) to give desired product 3.

3,3'-(Phenylmethylene)bis(1H-indole-5-carboxylic acid) 3a (Table 1, entry 2) Following the general procedure, 3a was obtained as a red solid. $93 \mathrm{mg}(91 \%)$; mp 212-214 ${ }^{\circ} \mathrm{C}$; IR $(\mathrm{KBr})\left(\mathrm{cm}^{-1}\right)$ 3424, 1680; ${ }^{1} \mathrm{H}$ NMR (400 MHz, DMSO-d 6 ): $\delta 5.98(\mathrm{~s}, 1 \mathrm{H}), 6.87(\mathrm{~s}, 2 \mathrm{H}), 7.20(\mathrm{t}, J=6.4 \mathrm{~Hz}, 1 \mathrm{H}), 7.30$ (t, $J=7.2 \mathrm{~Hz}, 2 \mathrm{H}), 7.36$ (d, $J=7.6 \mathrm{~Hz}, 2 \mathrm{H}), 7.41$ (d, $J=8.8 \mathrm{~Hz}, 2 \mathrm{H}), 7.69$ (dd, $J=8.4 \mathrm{~Hz}, 2 \mathrm{H}), 8.01$ (s, 2H), 11.2 (s, 2H), 12.3 (brs, 2H); ${ }^{13} \mathrm{C}$ NMR (100 MHz, DMSO-d 6 ): $\delta 111.3,119.3,120.8,121.8$, 122.4, 125.3, 126.1, 128.2, 139.1, 144.3, 168.3; MS (EI): $m / z(\%) 410\left(\mathrm{M}^{+}, 100\right)$; Anal. Calcd for $\mathrm{C}_{25} \mathrm{H}_{18} \mathrm{~N}_{2} \mathrm{O}_{4}$ 0.6 $\mathrm{H}_{2} \mathrm{O}$ : C, 71.28; H, 4.59; N, 6.65. Found: C, 71.22; H, 4.59; N, 6.37.

3,3'-(p-Tolylmethylene)bis(1H-indole-5-carboxylic acid) $3 \mathrm{~b}$ Following the general procedure, $\mathbf{3 b}$ was obtained as a red solid. $88 \mathrm{mg}(83 \%)$; mp 222-224 ${ }^{\circ} \mathrm{C}$; IR $(\mathrm{KBr})\left(\mathrm{cm}^{-1}\right) 3411,1682 ;{ }^{1} \mathrm{H}$ NMR (400 MHz, DMSO-d $\left.)_{6}\right): \delta 2.26(\mathrm{~s}, 3 \mathrm{H}), 5.93(\mathrm{~s}, 1 \mathrm{H}), 6.85(\mathrm{~d}, J=1.6 \mathrm{~Hz}, 2 \mathrm{H}), 7.10(\mathrm{~d}, J=8.0 \mathrm{~Hz}, 2 \mathrm{H})$, $7.23(\mathrm{~d}, J=8.0 \mathrm{~Hz}, 2 \mathrm{H}), 7.41(\mathrm{~d}, J=8.4 \mathrm{~Hz}, 2 \mathrm{H}), 7.69(\mathrm{dd}, J=8.8,1.6 \mathrm{~Hz}, 2 \mathrm{H}), 8.00(\mathrm{~d}, J=0.8 \mathrm{~Hz}$, 2H), 11.2 (s, 2H), 12.3 (brs, 2H); ${ }^{13} \mathrm{C}$ NMR (100 MHz, DMSO-d 6 ): $\delta$ 20.6, 111.3, 119.5, 120.8, 121.9, 122.3, 125.2, 126.1, 128.1, 128.8, 134.9, 139.2, 141.3, 168.3; MS(EI): $m / z(\%) 424\left(\mathrm{M}^{+}, 37.2\right), 161$ (100); Anal. Calcd for $\mathrm{C}_{26} \mathrm{H}_{20} \mathrm{~N}_{2} \mathrm{O}_{4} 0.6 \mathrm{H}_{2} \mathrm{O}: \mathrm{C}, 71.75 ; \mathrm{H}, 4.91 ; \mathrm{N}, 6.44$. Found: C, 71.80; H, 4.80; $\mathrm{N}, 6.35$.

3,3'-((4-Ethylphenyl)methylene)bis(1H-indole-5-carboxylic acid) $3 \mathrm{c}$ Following the general procedure, 3c was obtained as a red solid. $101 \mathrm{mg}(92 \%)$; mp 206-208 ${ }^{\circ} \mathrm{C}$; IR (KBr) $\left(\mathrm{cm}^{-1}\right) 3414$, 1679; ${ }^{1} \mathrm{H}$ NMR (400 MHz, DMSO-d 6 ): $\delta 1.16$ (t, $\left.J=7.6 \mathrm{~Hz}, 3 \mathrm{H}\right), 2.56$ (q, $J=7.6 \mathrm{~Hz}, 2 \mathrm{H}$ ), 5.94 (s, $1 \mathrm{H}), 6.87(\mathrm{~d}, J=2.0 \mathrm{~Hz}, 2 \mathrm{H}), 7.13(\mathrm{~d}, J=8.4 \mathrm{~Hz}, 2 \mathrm{H}), 7.27(\mathrm{~d}, J=8.0 \mathrm{~Hz}, 2 \mathrm{H}), 7.41(\mathrm{~d}, J=8.4 \mathrm{~Hz}$, 2H), $7.68(\mathrm{dd}, J=8.4,1.2 \mathrm{~Hz}, 2 \mathrm{H}), 8.01(\mathrm{~d}, J=0.8 \mathrm{~Hz}, 2 \mathrm{H}), 11.2(\mathrm{~s}, 2 \mathrm{H}), 12.3(\mathrm{brs}, 2 \mathrm{H}) ;{ }^{13} \mathrm{C}$ NMR (100 MHz, DMSO-d $\left.{ }_{6}\right): \delta 15.4,27.7,111.2,119.5,120.8,121.8,122.3,125.2,126.1,127.6,128.1$, 139.1, 141.2, 141.5, 168.3; MS(EI): $m / z(\%) 438\left(\mathrm{M}^{+}, 13.6\right), 161$ (100); Anal. Calcd for $\mathrm{C}_{27} \mathrm{H}_{22} \mathrm{~N}_{2} \mathrm{O}_{4}$ $0.6 \mathrm{H}_{2} \mathrm{O}: \mathrm{C}, 72.18 ; \mathrm{H}, 5.20 ; \mathrm{N}, 6.24$. Found: C, 72.25; H, 4.95; N, 6.18.

3,3'-((4-Methoxyphenyl)methylene)bis(1H-indole-5-carboxylic acid) 3d Following the general procedure, $\mathbf{3 d}$ was obtained as a red solid. $59 \mathrm{mg}(54 \%)$; $\mathrm{mp} 241-243{ }^{\circ} \mathrm{C}$; IR (KBr) $\left(\mathrm{cm}^{-1}\right) 3400,1681$; ${ }^{1} \mathrm{H}$ NMR (400 MHz, DMSO-d $\left.{ }_{6}\right): \delta 3.72(\mathrm{~s}, 3 \mathrm{H}), 5.92(\mathrm{~s}, 1 \mathrm{H}), 6.84(\mathrm{~d}, J=2.0 \mathrm{~Hz}, 2 \mathrm{H}), 6.86(\mathrm{~d}, J=8.8 \mathrm{~Hz}$, $2 \mathrm{H}), 7.26(\mathrm{~d}, J=8.8 \mathrm{~Hz}, 2 \mathrm{H}), 7.41(\mathrm{~d}, J=8.4 \mathrm{~Hz}, 2 \mathrm{H}), 7.69$ (dd, $J=8.4,1.6 \mathrm{~Hz}, 2 \mathrm{H}), 8.01(\mathrm{~s}, 2 \mathrm{H})$, 11.2 (s, 2H), 12.3 (brs, 2H); ${ }^{13} \mathrm{C}$ NMR (100 MHz, DMSO-d 6 ): $\delta 38.3,54.9,111.2,113.6,119.7,120.8$, $121.9,122.3,125.2,126.1,129.1,136.2,139.2,157.5,168.3$; MS(EI): $m / z(\%) 440\left(\mathrm{M}^{+}, 12.5\right), 161$ 
(100); Anal. Calcd for $\mathrm{C}_{26} \mathrm{H}_{20} \mathrm{~N}_{2} \mathrm{O}_{5}$ 0.7 $\mathrm{H}_{2} \mathrm{O}: \mathrm{C}, 68.93 ; \mathrm{H}, 4.76$; N, 6.18. Found: C, 68.92; H, 4.62; $\mathrm{N}, 6.05$.

3,3'-((3-Methoxyphenyl)methylene)bis(1H-indole-5-carboxylic acid) 3e Following the general procedure, 3e was obtained as a pale brown solid. $60 \mathrm{mg}(54 \%)$; mp 258-260 ${ }^{\circ} \mathrm{C}$; IR $(\mathrm{KBr})\left(\mathrm{cm}^{-1}\right)$ 3425, 3291, 1697; ${ }^{1} \mathrm{H}$ NMR (400 MHz, DMSO-d 6 ): $\delta 3.68(\mathrm{~s}, 3 \mathrm{H}), 5.96(\mathrm{~s}, 1 \mathrm{H}), 6.78(\mathrm{dd}, J=8.0$, $2.0 \mathrm{~Hz}, 1 \mathrm{H}), 6.85-6.95(\mathrm{~m}, 4 \mathrm{H}), 7.22(\mathrm{dd}, J=7.6,7.6 \mathrm{~Hz}, 1 \mathrm{H}), 7.42(\mathrm{~d}, J=8.4 \mathrm{~Hz}, 2 \mathrm{H}), 7.69$ (d, $J=$ $8.4 \mathrm{~Hz}, 2 \mathrm{H}), 8.02(\mathrm{~s}, 2 \mathrm{H}), 11.2(\mathrm{~s}, 2 \mathrm{H}), 12.3$ (brs, 2H); ${ }^{13} \mathrm{C}$ NMR (100 MHz, DMSO-d 6 ): $\delta$ 54.9, 111.0, 111.3, 114.5, 119.2, 120.7, 120.9, 121.9, 122.4, 125.3, 126.1, 129.2, 139.1, 146.0, 159.2, 168.4; MS (EI): $m / z(\%) 440\left(\mathrm{M}^{+}, 17.0\right), 161(100)$; Anal. Calcd for $\mathrm{C}_{26} \mathrm{H}_{20} \mathrm{~N}_{2} \mathrm{O}_{5} 0.5 \mathrm{H}_{2} \mathrm{O}: \mathrm{C}, 69.48 ; \mathrm{H}, 4.71 ; \mathrm{N}$, 6.23. Found: C, 69.50; H, 4.57; N, 6.20.

3,3'-((4-Fluorophenyl)methylene)bis(1H-indole-5-carboxylic acid) $3 f$ Following the general procedure, $\mathbf{3 f}$ was obtained as a red solid. $56 \mathrm{mg}(52 \%)$; $\mathrm{mp} 210-212^{\circ} \mathrm{C}$; IR $(\mathrm{KBr})\left(\mathrm{cm}^{-1}\right) 3428,1680$; ${ }^{1} \mathrm{H}$ NMR (400 MHz, DMSO-d 6 ): $\delta 6.02(\mathrm{~s}, 1 \mathrm{H}), 6.86(\mathrm{~d}, J=2.0 \mathrm{~Hz}, 2 \mathrm{H}), 7.12(\mathrm{dd}, J=8.8,8.8 \mathrm{~Hz}$, $2 \mathrm{H}), 7.38(\mathrm{dd}, J=8.8,5.6 \mathrm{~Hz}, 2 \mathrm{H}), 7.42(\mathrm{~d}, J=8.4 \mathrm{~Hz}, 2 \mathrm{H}), 7.69$ (dd, $J=8.4,1.6 \mathrm{~Hz}, 2 \mathrm{H}), 8.00$ (s, 2H), 11.2 (s, 2H), 12.3 (brs, 2H); ${ }^{13} \mathrm{C}$ NMR (100 MHz, DMSO-d 6 ): $\delta 38.3,111.3,114.9(J=22 \mathrm{~Hz})$, 119.3, 120.9, 121.8, 122.4, 125.3, 126.0, $130.0(J=7 \mathrm{~Hz}), 139.2,140.4(J=3 \mathrm{~Hz}), 160.6(J=242 \mathrm{~Hz})$,

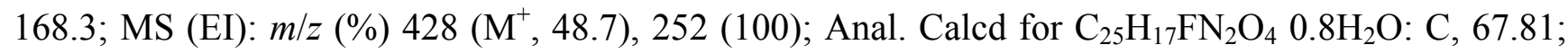
H, 4.23; N, 6.33. Found: C, 67.96; H, 4.12; N, 6.41.

3,3'-((4-Chlorophenyl)methylene)bis(1H-indole-5-carboxylic acid) $3 \mathrm{~g}$ Following the general procedure, $3 \mathrm{~g}$ was obtained as a pale brown solid. $75 \mathrm{mg}(67 \%)$; $\mathrm{mp} 211-213{ }^{\circ} \mathrm{C}$; IR $(\mathrm{KBr})\left(\mathrm{cm}^{-1}\right)$ 3435, 1679; ${ }^{1} \mathrm{H}$ NMR (400 MHz, DMSO-d 6 ): $\delta 6.03(\mathrm{~s}, 1 \mathrm{H}), 6.87(\mathrm{~d}, J=2.0 \mathrm{~Hz}, 2 \mathrm{H}), 7.36(\mathrm{~s}, 4 \mathrm{H})$, $7.42(\mathrm{~d}, J=8.4 \mathrm{~Hz}, 2 \mathrm{H}), 7.69(\mathrm{dd}, J=8.4,1.2 \mathrm{~Hz}, 2 \mathrm{H}), 8.00(\mathrm{~s}, 2 \mathrm{H}), 11.2$ (brs, 2H), 12.4 (brs, $2 \mathrm{H}) ;{ }^{13} \mathrm{C}$ NMR (100 MHz, DMSO-d $\left.{ }_{6}\right): \delta 38.4,111.3,118.9,120.9,121.7,122.4,125.4,126.0,128.2,130.1$, 130.6, 139.1, 143.4, 168.3; $\mathrm{MS}(\mathrm{EI}): \mathrm{m} / z(\%) 446\left(\mathrm{M}^{+}+2,17.3\right), 444\left(\mathrm{M}^{+}, 45.2\right), 252$ (100); Anal. Calcd for $\mathrm{C}_{25} \mathrm{H}_{17} \mathrm{ClN}_{2} \mathrm{O}_{4}$ 0.3 $\mathrm{H}_{2} \mathrm{O}$ : C, 66.69; H, 3.94; N, 6.22. Found: C, 66.56; H, 3.97; N, 6.20.

3,3'-(Thiophen-2-ylmethylene)bis(1H-indole-5-carboxylic acid) $\mathbf{3 h}$ Following the general procedure, $3 \mathbf{h}$ was obtained as a brown solid. $54 \mathrm{mg}(52 \%) ; \mathrm{mp} 228-230{ }^{\circ} \mathrm{C}$; IR $(\mathrm{KBr})\left(\mathrm{cm}^{-1}\right) 3392$, 1682; ${ }^{1} \mathrm{H}$ NMR (400 MHz, DMSO-d $)_{6}$ ): $\delta 6.29$ (s, 1H), 6.90-7.00 (m, 2H), $7.09(\mathrm{~s}, 2 \mathrm{H}), 7.34(\mathrm{dd}$, $J=4.8,1.2 \mathrm{~Hz}, 1 \mathrm{H}), 7.43$ (d, $J=8.8 \mathrm{~Hz}, 2 \mathrm{H}), 7.70$ (d, $J=8.8 \mathrm{~Hz}, 2 \mathrm{H}), 8.09$ (s, 2H), 11.3 (brs, 2H), 12.4 (brs, 2H); ${ }^{13} \mathrm{C}$ NMR (100 MHz, DMSO-d 6 ): $\delta 34.5,111.3,119.3,120.9,121.9,122.4,124.1$, 124.9, 125.0, 125.8, 126.6, 139.1, 148.8, 168.3; MS(EI): $m / z(\%) 416\left(\mathrm{M}^{+}, 18.7\right), 161$ (100); Anal. Calcd for $\mathrm{C}_{23} \mathrm{H}_{16} \mathrm{~N}_{2} \mathrm{O}_{4} \mathrm{~S} 1.0 \mathrm{H}_{2} \mathrm{O}$ : C, 63.58; H, 4.18; N, 6.45. Found: C, 63.86; H, 4.08; N, 6.18.

3,3'-(Naphthalen-2-ylmethylene)bis(1H-indole-5-carboxylic acid) $3 \mathbf{i}$ Following the general procedure, $3 \mathbf{i}$ was obtained as a pale brown solid. $110 \mathrm{mg}(96 \%) ; \mathrm{mp} 222-224{ }^{\circ} \mathrm{C}$; IR $(\mathrm{KBr})\left(\mathrm{cm}^{-1}\right)$ 3419, 1676; ${ }^{1} \mathrm{H}$ NMR (400 MHz, DMSO-d $)$ ) $\delta 6.17$ (s, 1H), $6.91(\mathrm{~s}, 2 \mathrm{H}), 7.40-7.48(\mathrm{~m}, 4 \mathrm{H}), 7.56(\mathrm{~d}$, $J=8.4 \mathrm{~Hz}, 1 \mathrm{H}), 7.69$ (d, $J=8.8 \mathrm{~Hz}, 2 \mathrm{H}), 7.76-7.88$ (m, 4H), 8.05 (s, 2H), 11.2 (brs, 2H), 12.3 (brs, $2 \mathrm{H}) ;{ }^{13} \mathrm{C}$ NMR (100 MHz, DMSO-d 6 ): $\delta 111.3,119.2,120.9,121.8,122.4,125.5,125.9,126.0,126.1$, $127.4,127.5,127.6,127.7,131.8,133.1,139.2,142.0,168.3$; MS (EI): $m / z(\%) 460\left(\mathrm{M}^{+}, 98.1\right), 161$ (100); Anal. Calcd for $\mathrm{C}_{29} \mathrm{H}_{20} \mathrm{~N}_{2} \mathrm{O}_{4} 1.0 \mathrm{H}_{2} \mathrm{O}: \mathrm{C}, 72.79 ; \mathrm{H}, 4.63 ; \mathrm{N}, 5.85$. Found: C, 72.75; H, 4.49; N, 5.75 .

3,3'-(Phenylmethylene)bis(1H-indole-4-carboxylic acid) $3 \mathbf{j}$ Following the general procedure, 3j was obtained as a red solid. $53 \mathrm{mg}(52 \%)$; mp 266-268 ${ }^{\circ} \mathrm{C}$; IR $(\mathrm{KBr})\left(\mathrm{cm}^{-1}\right) 3413,3063,1682 ;{ }^{1} \mathrm{H}$ 
NMR (400 MHz, DMSO-d $): \delta 6.24(\mathrm{~s}, 2 \mathrm{H}), 6.86(\mathrm{~s}, 1 \mathrm{H}), 7.00(\mathrm{~d}, J=4.0 \mathrm{~Hz}, 2 \mathrm{H}), 7.05-7.15(\mathrm{~m}, 4 \mathrm{H})$, $7.18(\mathrm{dd}, J=8.0,8.0 \mathrm{~Hz}, 2 \mathrm{H}), 7.31(\mathrm{~d}, J=8.0 \mathrm{~Hz}, 2 \mathrm{H}), 7.53(\mathrm{~d}, J=8.0 \mathrm{~Hz}, 2 \mathrm{H}), 11.0(\mathrm{~d}, J=4.0 \mathrm{~Hz}$, 2H), 12.0 (brs, 2H); ${ }^{13} \mathrm{C}$ NMR (100 MHz, DMSO-d 6 ): $\delta 41.4,115.0,119.6,120.3,121.0,123.4,125.1$, 126.2, 126.9, 127.5, 129.2, 138.1, 146.7, 168.9; MS(ESI): $m / z(\%) 410\left(\mathrm{M}^{+}\right)$; Anal. Calcd for $\mathrm{C}_{25} \mathrm{H}_{18} \mathrm{~N}_{2} \mathrm{O}_{4}$ 0.3 $\mathrm{H}_{2} \mathrm{O}: \mathrm{C}, 72.21 ; \mathrm{H}, 4.51 ; \mathrm{N}, 6.74$. Found: $\mathrm{C}, 72.29 ; \mathrm{H}, 4.44 ; \mathrm{N}, 6.62$.

3,3'-(Phenylmethylene)bis(1H-indole-6-carboxylic acid) 3k Following the general procedure, 3k was obtained as a pale brown solid. $62 \mathrm{mg}(60 \%)$; mp 280-283 ${ }^{\circ} \mathrm{C}$; IR $(\mathrm{KBr})\left(\mathrm{cm}^{-1}\right) 3376,3011,1672$; ${ }^{1} \mathrm{H}$ NMR (400 MHz, DMSO-d $\left.{ }_{6}\right): \delta 5.91(\mathrm{~s}, 1 \mathrm{H}), 7.07$ (d, $\left.J=2.0 \mathrm{~Hz}, 2 \mathrm{H}\right), 7.19(\mathrm{t}, J=7.2 \mathrm{~Hz}, 1 \mathrm{H})$, $7.26-7.40(\mathrm{~m}, 7 \mathrm{H}), 7.49(\mathrm{dd}, J=8.4,1.2 \mathrm{~Hz}, 2 \mathrm{H}), 8.02(\mathrm{~s}, 2 \mathrm{H}), 11.2(\mathrm{brs}, 2 \mathrm{H}) ;{ }^{13} \mathrm{C} \mathrm{NMR}(100 \mathrm{MHz}$, DMSO-d $\left.{ }_{6}\right): \delta 113.7,118.4,118.6,119.3,123.2,126.0,127.3,128.2,129.7,135.9,144.4,168.4$; MS (EI): $m / z(\%) 410\left(\mathrm{M}^{+}, 56.5\right), 161$ (100); Anal. Calcd for $\mathrm{C}_{25} \mathrm{H}_{18} \mathrm{~N}_{2} \mathrm{O}_{4} 0.4 \mathrm{H}_{2} \mathrm{O}: \mathrm{C}, 71.90 ; \mathrm{H}, 4.54 ; \mathrm{N}$, 6.71. Found: C, 71.81; H, 4.50; N, 6.47.

3,3'-(Phenylmethylene)bis(1H-indole-7-carboxylic acid) 31 Following the general procedure, 31 was obtained as a pink solid. $78 \mathrm{mg}(76 \%)$; $\mathrm{mp} 198-200{ }^{\circ} \mathrm{C}$; IR $(\mathrm{KBr})\left(\mathrm{cm}^{-1}\right) 3453,3035,1668 ;{ }^{1} \mathrm{H}$ NMR (400 MHz, DMSO-d $)$ ): $\delta 5.94(\mathrm{~s}, 1 \mathrm{H}), 6.79(\mathrm{~s}, 2 \mathrm{H}), 6.99(\mathrm{dd}, J=7.6,7.6 \mathrm{~Hz}, 1 \mathrm{H}), 7.20$ (t, $J=7.2 \mathrm{~Hz}, 1 \mathrm{H}), 7.30(\mathrm{dd}, J=7.2,7.2 \mathrm{~Hz}, 2 \mathrm{H}), 7.35$ (d, $J=7.2 \mathrm{~Hz}, 2 \mathrm{H}), 7.55$ (d, $J=7.6 \mathrm{~Hz}, 2 \mathrm{H}), 7.72$ (d, $J=7.6 \mathrm{~Hz}, 2 \mathrm{H}), 10.9$ (brs, 2H); ${ }^{13} \mathrm{C}$ NMR (100 MHz, DMSO-d 6 ): $\delta 113.5,117.9,118.3,123.9$, 124.5, 125.3, 126.1, 128.1, 128.2, 128.3, 135.3, 144.3, 168.0; MS (EI): $m / z(\%) 410\left(\mathrm{M}^{+}, 100\right)$; Anal. Calcd for $\mathrm{C}_{25} \mathrm{H}_{18} \mathrm{~N}_{2} \mathrm{O}_{4}$ 0.7 $\mathrm{H}_{2} \mathrm{O}: \mathrm{C}, 70.98 ; \mathrm{H}, 4.62 ; \mathrm{N}, 6.62$. Found: $\mathrm{C}, 70.86 ; \mathrm{H}, 4.55 ; \mathrm{N}, 6.51$.

3,3'-(Phenylmethylene)bis(1H-indole-2-carboxylic acid) $\mathbf{3 m}$ Following the general procedure, 3m was obtained as a purple solid. $46 \mathrm{mg}(45 \%)$; mp 231-234 ${ }^{\circ} \mathrm{C}$; IR (KBr) $\left(\mathrm{cm}^{-1}\right) 3390,3063,1677$; ${ }^{1} \mathrm{H}$ NMR (400 MHz, DMSO-d 6 ): $\delta 6.50(\mathrm{~d}, J=8.0 \mathrm{~Hz}, 2 \mathrm{H}), 6.61(\mathrm{dd}, J=8.0,8.0 \mathrm{~Hz}, 2 \mathrm{H}), 7.05-7.10$ (m, 4H), 7.20-7.25 (m, 3H), 7.39 (d, $J=8.4 \mathrm{~Hz}, 2 \mathrm{H}), 7.46$ (s, 1H), 11.6 (brs, 2H); ${ }^{13} \mathrm{C}$ NMR (100 MHz, DMSO-d $\left.{ }_{6}\right): \delta 112.6,119.0,121.8,123.4,123.6,125.2,125.8,127.2,127.9,128.7,136.0,144.7,162.9$; MS (EI): $m / z(\%) 410\left(\mathrm{M}^{+}, 15.5\right), 252$ (100); Anal. Calcd for $\mathrm{C}_{25} \mathrm{H}_{18} \mathrm{~N}_{2} \mathrm{O}_{4} \bullet 1.0 \mathrm{H}_{2} \mathrm{O}: \mathrm{C}, 70.08 ; \mathrm{H}, 4.71$; N, 6.54. Found: C, 69.85; H, 4.59; N, 6.24.

3-Benzyl-1H-indole-5-carboxylic acid 4a (Table 1, entry 1) Following the general procedure, 4a was obtained as an off-white solid. $38 \mathrm{mg}(15 \%)$; mp $184-186^{\circ} \mathrm{C}$; IR $(\mathrm{KBr})\left(\mathrm{cm}^{-1}\right) 3442,2966,2640$, 1673; ${ }^{1} \mathrm{H}$ NMR (400 MHz, DMSO-d ${ }_{6}$ ): $\delta 4.08(\mathrm{~s}, 2 \mathrm{H}), 7.10-7.20(\mathrm{~m}, 1 \mathrm{H}), 7.20-7.35(\mathrm{~m}, 5 \mathrm{H}), 7.39(\mathrm{~d}$, $J=8.8 \mathrm{~Hz}, 1 \mathrm{H}), 7.68(\mathrm{~d}, J=8.8 \mathrm{~Hz}, 1 \mathrm{H}), 8.09$ (s, $1 \mathrm{H}), 11.2$ (brs, $2 \mathrm{H}), 12.3$ (brs, 2H); ${ }^{13} \mathrm{C} \mathrm{NMR}(100 \mathrm{MHz}$, DMSO-d $\left.{ }_{6}\right): \delta 30.7,111.1,115.2,120.8,121.2,122.3,124.8,125.7,126.5,128.2,128.3,138.9,141.4$, 168.3; MS (EI): $m / z(\%) 251\left(\mathrm{M}^{+}, 100\right)$; $\mathrm{HRMS}(\mathrm{EI}): m / z$ calcd for $\mathrm{C}_{16} \mathrm{H}_{13} \mathrm{NO}_{2}\left[\mathrm{M}^{+}\right], 251.0946$ found 251.0944 .

\section{Conclusions}

In summary, we have demonstrated a novel method for the direct construction of $\mathrm{C}-\mathrm{C}$ bonds of unprotected indole carboxylic acid $\mathbf{1}$ and various benzyl alcohols $\mathbf{2}$ by Pd-catalyzed domino reactions in water. In our $\left(\eta^{3}\right.$-benzyl)palladium system, the domino reactions achieved $\mathrm{C}-\mathrm{H}$ activation of the C3-position of indole, nucleophilic attack of indoles to hydrated benzyl alcohols, and benzylic $\mathrm{C}-\mathrm{H}$ functionalization. Notably, water activated unreactive molecules such as hydroxyl groups and stabilized hydroxide ions by hydration, followed by formation of activated $\mathrm{Pd}(\mathrm{II})$ cation species. 


\section{Conflict of Interest}

The authors declare no conflict of interest.

\section{References}

1. Ueno, S.; Komiya, S.; Tanaka, T.; Kuwano, R. Intramolecular SN'-Type Aromatic Substitution of Benzylic Carbonates at their Para-Position. Org. Lett. 2012, 14, 338-341.

2. Xie, P.; Xie, Y.; Qian, B.; Zhou, H.; Xia, C.; Huang, H. Palladium-Catalyzed Oxidative Carbonylation of Benzylic C-H Bonds via Nondirected $\mathrm{C}\left(\mathrm{sp}^{3}\right)-\mathrm{H}$ Activation. J. Am. Chem. Soc. 2012, 134, 9902-9905.

3. Trost, B.M.; Czabaniuk, L.C. Benzylic Phosphates as Electrophiles in the Palladium-Catalyzed Asymmetric Benzylation of Azlactones. J. Am. Chem. Soc. 2012, 134, 5778-5781.

4. Peng, B.; Zhang, S.; Yu, X.; Feng, X.; Bao, M. Nucleophilic Dearomatization of Chloromethyl Naphthalene Derivatives via $\eta^{3}$-Benzylpalladium Intermediates: A New Strategy for Catalytic Dearomatization. Org. Lett. 2011, 13, 5402-5405.

5. Marquard, S.L.; Hartwig, J.F. C( $\left(\mathrm{sp}^{3}\right)-\mathrm{O}$ Bond-forming reductive elimination of ethers from bisphosphine-ligated benzylpalladium(II) aryloxide complexes. Angew. Chem. Int. Ed. 2011, 50, 7119-7123.

6. Marquard, S.L.; Rosenfeld, D.C.; Hartwig, J.F. C( $\left(\mathrm{sp}^{3}\right)-\mathrm{N}$ Bond-Forming Reductive Elimination of Amines: Reactions of Bisphosphine-Ligated Benzylpalladium(II) Diarylamido Complexes. Angew. Chem. Int. Ed. 2010, 49, 793-796.

7. Trost, B.M.; Czabaniuk, L.C. Palladium-Catalyzed Asymmetric Benzylation of 3-Aryl Oxindoles. J. Am. Chem. Soc. 2010, 132, 15534-15536.

8. Kuwano, R. Catalytic transformations of benzylic carboxylates and carbonates. Synthesis 2009, 1049-1061.

9. Kuwano, R.; Konda, Y.; Matsuyama, Y. Palladium-Catalyzed Nucleophilic Benzylic Substitutions of Benzylic Esters. J. Am. Chem. Soc. 2003, 125, 12104-12105.

10. Zhou, B.; Yang, J.; Li, M.; Gu, Y. Gluconic acid aqueous solution as a sustainable and recyclable promoting medium for organic reactions. Green Chem. 2011, 13, 2204-2211.

11. Shirakawa, S.; Kobayashi, S. Surfactant-Type Bronsted Acid Catalyzed Dehydrative Nucleophilic Substitutions of Alcohols in Water. Org. Lett. 2007, 9, 311-314.

12. Bisaro, F.; Prestat, G.; Vitale, M.; Poli, G. Alkylation of active methylenes via benzhydryl cations. Synlett 2002, 1823-1826.

13. Yu, D.-G.; Wang, X.; Zhu, R.-Y.; Luo, S.; Zhang, X.-B.; Wang, B.-Q.; Wang, L.; Shi, Z.-J. Direct Arylation/Alkylation/Magnesiation of Benzyl Alcohols in the Presence of Grignard Reagents via Ni-, Fe-, or Co-Catalyzed sp ${ }^{3}$ C-O Bond Activation. J. Am. Chem. Soc. 2012, 134, 14638-14641.

14. Ohshima, T.; Nakahara, Y.; Ipposhi, J.; Miyamotob, Y.; Mashima, K. Direct substitution of the hydroxy group with highly functionalized nitrogen nucleophiles catalyzed by $\mathrm{Au}(\mathrm{III})$. Chem. Commun. 2011, 8322-8324.

15. Hirashita, T.; Kuwahara, S.; Okochi, S.; Tsuji, M.; Araki, S. Direct benzylation and allylic alkylation in high-temperature water without added catalysts. Tetrahedron Lett. 2010, 51, 1847-1851. 
16. Cozzi, P.G.; Zoli, L. A rational approach towards the nucleophilic substitutions of alcohols "on water". Angew. Chem. Int. Ed. 2008, 47, 4162-4166.

17. Cozzi, P.G.; Zoli, L. Nucleophilic substitution of ferrocenyl alcohols "on water". Green Chem. 2007, 9, 1292-1295.

18. Liu, C.; Zhang, H.; Shi, W.; Lei, A. Bond formations between two nucleophiles: Transition metal catalyzed oxidative cross-coupling reactions. Chem. Rev. 2011, 111, 1780-1824.

19. Bras, J.L.; Muzart, J. Intermolecular Dehydrogenative Heck Reactions. Chem. Rev. 2011, 111, 1170-1214.

20. Yeung, C.S.; Dong, V.M. Catalytic Dehydrogenative Cross-Coupling: Forming Carbon-Carbon Bonds by Oxidizing Two Carbon-Hydrogen Bonds. Chem. Rev. 2011, 111, 1215-1292.

21. Lyons, T.W.; Sanford, M.S. Palladium-Catalyzed Ligand-Directed C-H Functionalization Reactions. Chem. Rev. 2010, 110, 1147-1169.

22. Sun, C.-L.; Li, B.-J.; Shi, Z.-J. Pd-catalyzed oxidative coupling with organometallic reagents via C-H activation. Chem. Commun. 2010, 677-685.

23. Chen, X.; Engle, K.M.; Wang, D.-H.; Yu, J.-Q. Palladium(II)-Catalyzed C-H Activation/C-C Cross-Coupling Reactions: Versatility and Practicality. Angew. Chem. Int. Ed. 2009, 48, 5094-5115.

24. Ackermann, L.; Vicente, R.; Kapdi, A.R. Transition metal-catalyzed direct arylation of (hetero)arenes by C-H bond cleavage. Angew. Chem. Int. Ed. 2009, 48, 9792-9826.

25. Hikawa, H.; Yokoyama, Y. Pd-catalyzed C-H activation in water: synthesis of bis(indolyl)methanes from indoles and benzyl alcohols. RSC Adv. 2013, 3, 1061-1064.

26. Hikawa, H.; Ino, Y.; Suzuki, H.; Yokoyama, Y. Pd-Catalyzed Benzylic C-H Amidation with Benzyl Alcohols in Water: A Strategy To Construct Quinazolinones. J. Org. Chem. 2012, 77, 7046-7051.

27. Hikawa, H.; Yokoyama, Y. Palladium-catalyzed S-benzylation of unprotected mercaptobenzoic acid with benzyl alcohols in water. Org. Biomol. Chem. 2012, 10, 2942-2945.

28. Hikawa, H.; Yokoyama, Y. Palladium-Catalyzed Benzylation of Unprotected Anthranilic Acids with Benzyl Alcohols in Water. Org. Lett. 2011, 13, 6512-6515.

29. Hikawa, H.; Yokoyama, Y. Palladium-catalyzed mono- $N$-allylation of unprotected anthranilic acids with allylic alcohols in aqueous media. J. Org. Chem. 2011, 76, 8433-8439.

30. Hikawa, H.; Yokoyama, Y. Palladium-catalyzed mono- $N$-allylation of unprotected amino acids with 1,1-dimethylallyl alcohol in water. Org. Biomol. Chem. 2011, 9, 4044-4050.

31. Yokoyama, Y.; Hikawa, H.; Mitsuhashi, M.; Uyama, A.; Hiroki, Y.; Murakami, Y. Total synthesis without protection: three-step synthesis of optically active clavicipitic acids by a biomimetic route. Eur. J. Org. Chem. 2004, 1244-1253.

32. Yokoyama, Y.; Hikawa, H.; Mitsuhashi, M.; Uyama, A.; Murakami, Y. Syntheses without protection: a three-step synthesis of optically active clavicipitic acid by utilizing biomimetic synthesis of 4-bromotryptophan. Tetrahedron Lett. 1999, 40, 7803-7806.

33. Afagh, N.A.; Yudin, A.K. Chemoselectivity and the Curious Reactivity Preferences of Functional Groups. Angew. Chem. Int. Ed. 2010, 49, 262-310.

34. Basset, J.-M.; Bouchu, D.; Godard, G.; Karame', I.; Kuntz, E.; Lefebvre, L.; Legagneux, N.; Lucas, C.; Michelet, D.; Tommasino, J.B. Heterolytic Splitting of Allylic Alcohols with 
Palladium(0)-TPPTS in Water. Stabilities of the Allylphosphonium Salt of TPPTS and of the Ionic Complex [Pd( $\eta^{3}$-allyl)(TPPTS $\left.)_{2}\right]^{+}$. Organometallics 2008, 27, 4300-4309.

35. Potavathri, S.P.; Pereira, K.C.; Gorelsky, S.I.; Pike, A.; LeBris, A.P.; DeBoef, B. Regioselective Oxidative Arylation of Indoles Bearing $N$-Alkyl Protecting Groups: Dual C-H Functionalization via a Concerted Metalation-Deprotonation Mechanism. J. Am. Chem. Soc. 2010, 132, 14676-14681.

36. Zhao, J.; Zhang, Y.; Cheng, K. Palladium-Catalyzed Direct C-2 Arylation of Indoles with Potassium Aryltrifluoroborate Salts. J. Org. Chem. 2008, 73, 7428-7431.

37. Lane, B.S.; Brown, M.A.; Sames, D. Direct Palladium-Catalyzed C-2 and C-3 Arylation of Indoles: A Mechanistic Rationale for Regioselectivity. J. Am. Chem. Soc. 2005, 127, 8050-8057.

38. Grimster, N.P.; Gauntlett, C.; Godfrey, C.R.A.; Gaunt, M.J. Palladium-catalyzed intermolecular alkenylation of indoles by solvent-controlled regioselective $\mathrm{C}-\mathrm{H}$ functionalization. Angew. Chem. Int. Ed. 2005, 44, 3125-3129.

39. Yokogi, M.; Kuwano, R. Use of acetate as a leaving group in palladium-catalyzed nucleophilic substitution of benzylic esters. Tetrahedron Lett. 2007, 48, 6109-6112.

40. Kuwano, R.; Yokogi, M. Cross-coupling of benzylic acetates with arylboronic acids: One-pot transformation of benzylic alcohols to diarylmethanes. Chem. Commun. 2005, 5899-5901.

41. Carter, M.D.; Hadden, M.; Weaver, D.F.; Jacobo, S.M.H.; Lu, E. Preparation of bis-hetero/aryls, particularly bis-indoles, for treatment of protein folding disorders. WO2006/125324, 30 November 2006.

42. Nobuta, T.; Fujiya, A.; Tada, N.; Miura, T.; Itoh, A. One-pot synthesis of bisindolylmethanes from benzyl alcohols and indoles using catalytic iodine and molecular oxygen under visible light irradiation. Synlett 2012, 2975-2979.

43. Anilkumar, G.N.; Lesburg, C.A.; Selyutin, O.; Rosenblum, S.B.; Zeng, Q.; Jiang, Y.; Chan, T.-Y.; $\mathrm{Pu}, \mathrm{H}$; Vaccaro, H.; Wang, L.; et al. Novel HCV NS5B polymerase inhibitors: Discovery of indole 2-carboxylic acids with C3-heterocycles. Bioorg. Med. Chem. Lett. 2011, 21, 5336-5341.

44. Bovens, S.; Elfringhoff, S.A.; Kaptur, M.; Reinhardt, D.; Schäfers, M.; Lehr, M. 1-(5-Carboxyindol-1-yl)propan-2-one Inhibitors of Human Cytosolic Phospholipase A2 $\alpha$ : Effect of Substituents in Position 3 of the Indole Scaffold on Inhibitory Potency, Metabolic Stability, Solubility, and Bioavailability. J. Med. Chem. 2010, 53, 8298-8308.

45. Ludwig, J.; Bovens, S.; Brauch, C.; Elfringhoff, S.A.; Lehr, M. Design and synthesis of 1-Indol-1yl-propan-2-ones as inhibitors of human cytosolic phospholipase A2 $\alpha$. J. Med. Chem. 2006, 49, 2611-2620.

46. Mazzei, M.; Miele, M.; Nieddu, E.; Barbieri, F.; Bruzzo, C.; Alama, A. Unsymmetrical methylene derivatives of indoles as antiproliferative agents. Eur. J. Med. Chem. 2001, 36, 915-923.

47. Kinoshita, H.; Shinokubo, H.; Oshima, K. Water Enables Direct Use of Allyl Alcohol for Tsuji-Trost Reaction without Activators. Org. Lett. 2004, 6, 4085-4088.

(C) 2013 by the authors; licensee MDPI, Basel, Switzerland. This article is an open access article distributed under the terms and conditions of the Creative Commons Attribution license (http://creativecommons.org/licenses/by/3.0/). 